\section{LEARNING CORNER}

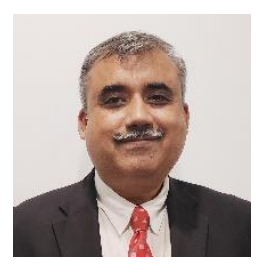

SECTION EDITOR

Aamir Hameed Khan

Aga Khan University Hospital, Karachi,

Pakistan
Dr. Amir is the Badruddin Hassan Ali Jeeva Professor of Medicine and Consultant Cardiologist, Chief Heart Lung and Vascular Service line, Director Clinical Cardiac Electrophysiology Fellowship Program at Aga Khan University Hospital, Karachi.

\title{
THE ROLE OF DIGOXIN IN THE SUCCESSFUL TERMINATION OF A RESISTANT MATERNAL ATYPICAL AV NODAL REENTRY TACHYCARDIA
}

\author{
Naheed Feroz Ali', Saba Salim Qazi ${ }^{2}$, Intisar Ahmed ${ }^{1}$, Muhammad Ahmed Tamiz ${ }^{1}$, \\ Asfandiar Ali ${ }^{1}$, Aamir Hameed Khan ${ }^{1}$ \\ ${ }^{1}$ Aga Khan University, Karachi Pakistan, ${ }^{2}$ Ziauddin Medical University, Karachi, Pakistan
}

\section{INTRODUCTION}

During gestation the blood volume increases by an average of $50 \%$. Blood pressure usually falls, reaching a nadir in mid-pregnancy. The systolic pressure usually returns to near pre-gestational levels by term. ${ }^{1}$ With the rise in blood volume the cardiac output also rises by about $50 \%$ with a steep increase from the latter half of the first trimester. The increase in blood volume can cause atrial stretch that may be significant for arrhythmogenesis. Later in pregnancy a rise in heart rate, on average 20 beats per minute, accounts for further enhancement in cardiac output. ${ }^{2}$ The hormonal changes that occur during gestation may also exert a proarrhythmic effect on myocardial tissue. ${ }^{3}$ All of the this is accompanied by an increase in the sympathetic outflow, which also contributes towards arrhythmogenesis. ${ }^{4}$ Arrhythmias that cause prolonged maternal hypotension and threaten placental perfusion, require urgent treatment. Treatment may be extended to prevent distressing symptoms. The decision to use a particular therapy must take into account the risks of that therapy to both the mother and child. ${ }^{5}$ Supraventricular tachycardia (SVT) occurs more often during gestation. ${ }^{6}$ Fetal safety should be the paramount consideration during the first trimester ( $5^{\text {th }}$ to $10^{\text {th }}$ week) due to organ formation. ${ }^{7}$ Drug safety from this point of view should also be of utmost concern. Drugs classifications have divided drugs that are considered relatively safe during the different periods of gestation. Some drugs that are used safely during non-gestational SVT may not be considered during gestation, especially during the organ formation stage, while others that have fallen out of favor for the usual SVT treatment, may work very well during challenging cases.

Here, we describe the case of a patient during her gestational period with resistant tachycardia that failed front line treatment options and was treated using an older agent.

\section{CASE DESCRIPTION}

A 39-year-old multiparous female with 28 weeks of gestation presented to the emergency department (ED) with sudden onset of palpitations lasting 2 hours. The palpitations were happening on and off for two days and were not associated with chest pain, shortness of breath, syncope, or orthopnea. The patient had no known previous medical history. However, she had a previous history of two spontaneous abortions and had undergone three lower segment Cesarian Section (LSCS) with no maternal or fetal complications. The patient had experienced a similar episode of SVT seven years ago during her last pregnancy, which had been managed with propranolol infusion. The patient was currently experiencing a "Vanishing twin syndrome", 8 where she had a spontaneous abortion of one of the twins at 20 weeks of gestation. Since then, the patient's palpitations had accentuated even though she was on bisoprolol $5 \mathrm{mg}$.

On arrival in the emergency department (ED), electrocardiogram (EKG) revealed a narrow complex tachycardia (Figure 1) The pulse rate was 155 beats/minutes with hemodynamic stability (BP: $135 / 55 \mathrm{~mm}$ of $\mathrm{Hg}$ ). Carotid sinus massage was performed with no effect on the tachycardia. A quick 
echocardiogram revealed an ejection fraction of $60 \%$, and did not show structural abnormality. With an urgent cardiac consultation, the patient was given IV adenosine via a cubital vein cannula with saline push (total of $24 \mathrm{mg}$ in escalating doses). The tachycardia would terminate momentarily and restart again. Subsequently, intravenous metoprolol $5 \mathrm{mg}$ aliquots were given with partial effect, additionally, verapamil $5 \mathrm{mg}$ aliquots were also given. The tachycardia remained resistant to treatment and was sustained at the rate of 145 beats/minute. Physical examination was unremarkable other than for tachycardia. Her baseline investigations ruled out anemia and showed a euthyroid state. The patient was immediately shifted to the coronary care unit (CCU) for further management.

The 12 lead ECG (Figure 1A) shows a narrow complex tachycardia with a $\mathrm{P}$ wave that was clearly identified. There was 1:1 conduction revealing a $\mathrm{P}$ wave for each narrow complex QRS. As per the narrow complex tachycardia algorithm the next interval to measure is the RP interval. The RP interval (Figure 1B) was longer as compared to the next PR interval. The differential diagnosis for a long RP tachycardia is sinus/atrial tachycardia, atypical AV nodal reentry tachycardia (AVNRT) and permanent junction reciprocating tachycardia (PJRT). Atrial tachycardia (unless reentrant) starts gradually, and the initiating $\mathrm{P}$ wave is different from the tachycardia $\mathrm{P}$ waves. In our case the $P$ wave that starts the tachycardia (Figure 2A) is different from the tachycardia $\mathrm{P}$ wave. Atrial tachycardia does not terminate on a $\mathrm{P}$ wave. If a narrow complex tachycardia terminates on a $\mathrm{P}$ wave it is most likely to be an SVT. PJRTs are macro AV reentrant tachycardias dependent on a decremental accessory pathway that only conducts retrogradely. These are incessant tachycardias and are discovered in the pediatric population. In our patient continuous ECG lead II rhythm strip (Figure 2A) shows spontaneous termination of the tachycardia on a $\mathrm{P}$ wave, which practically rules out atrial tachycardia. So, our working diagnosis was atypical AVNRT, which is a relatively rare presentation of SVT. It being resistant to mainline therapy and during the gestational period made management challenging.

The atypical AVNRT spontaneously terminated after a couple of hours of arrival in the CCU, but soon after, the patient again complained of palpitations and the EKG showed the same SVT. Repeat doses of intravenous verapamil $10 \mathrm{mg}$ (two doses of $5 \mathrm{mg}$ ), metoprolol 5mg, and adenosine $(18 \mathrm{mg})$ were administered with no effect. After due consultation with the Electrophysiology service, a digoxin loading protocol was initiated $(0.5 \mathrm{mg}$ intravenous stat dose followed by $0.25 \mathrm{mg}$ every 4 hours to make $1 \mathrm{mg}$ and then $0.25 \mathrm{mg}$ tablet per day) with monitoring of creatinine, electrolytes, and digoxin levels. The patient came into sinus rhythm and remained stable thereafter. The patient was discharged home on $0.25 \mathrm{mg}$ of digoxin once a day and metoprolol 50mg twice per day. In a follow-up visit after a week, both the mother and the baby were stable. The fetal cardiac monitoring was satisfactory, so metoprolol dose was escalated.

\section{DISCUSSION}

Sustained SVT is more frequent in pregnancy, which enhances the risk of maternal mortality during pregnancy. AVNRT is the most common type of SVT (60\% of the cases), approximately $6.4 \%$ of patients with AVNRT present with delayed retrograde atrial activation, and these arrhythmias are characterized as atypical variants. ${ }^{9}$ Atypical AVNRT shows a negative $\mathrm{P}$ wave before $\mathrm{QRS}$ complex, $\mathrm{RP}^{\prime} / \mathrm{P}^{\prime} \mathrm{R}>0.75$ indicating long RP tachycardia, with an episode of sudden onset and abrupt termination of tachycardia on $\mathrm{A}$ (P-wave) (Figure 2A and 2B). ${ }^{10}$

The exact mechanism of atypical AVNRT occurrence in pregnancy is unknown. However, the possible causes might be the combination of an increase in autonomic response, significant hemodynamic and hormonal change that may exacerbate pre-existing arrhythmias or provoke arrhythmias for the first-time during pregnancy. This change in cardiovascular physiology usually begins at 8 weeks and peaks at 32 weeks of gestation. ${ }^{7}$

Vagal maneuvers form the first line therapeutic maneuvers with only $3 \%$ success rate. ${ }^{11}$ Drug therapy may be attempted if SVT is hemodynamically well tolerated. The medical management of SVT in pregnancy is quite comparable to the non-pregnant population. However, it differs slightly based on the trimester of pregnancy. Beta-blockers, other than atenolol and calcium channel blockers are relatively safe and effective in managing SVT with minimal fetal and maternal risk. ${ }^{12}$ Verapamil can get into the fetal circulation and cause fetal bradycardia in the first trimester and should be used with care. Adenosine remains the first line of treatment due to its ultrashort half-life, safety, and efficacy. ${ }^{13}$ Digoxin has been less used as first line therapy but has a good safety profile and can be used effectively in cases that are resistant to the first line therapy. ${ }^{13}$ Direct current cardioversion (DCCV), although rarely used, is highly efficacious in terminating sustained maternal SVT causing hemodynamic instability and in drug-refractory patients after ensuring fetal viability. DCCV has been 
proven to be safe in all stages in life-threatening circumstances. Synchronized DCCV is typically administered at 50 to 400 joules. ${ }^{14}$ In cases that are incessant and resistant to all the above therapies, then invasive electrophysiologic study and radiofrequency ablation (EPS-RFA) can be considered. ${ }^{15}$ Although, it requires $\mathrm{X}$ ray for visualization in most conventional cases, it can also be offered with fetal shield during the procedure, in the later half of pregnancy. ${ }^{16}$ Three- dimensional mapping systems can also be used as the navigational modality minimizing or obviating the need for any exposure to ionizing radiation altogether. EPS-RFA has proven success and it has significantly reduced the mortality rate in SVT patients. ${ }^{17}$ In the very resistant cases, where EPS-RFA is not available then amiodarone may be used for the acute treatment but used in special cases only and as a last resort. ${ }^{1}$

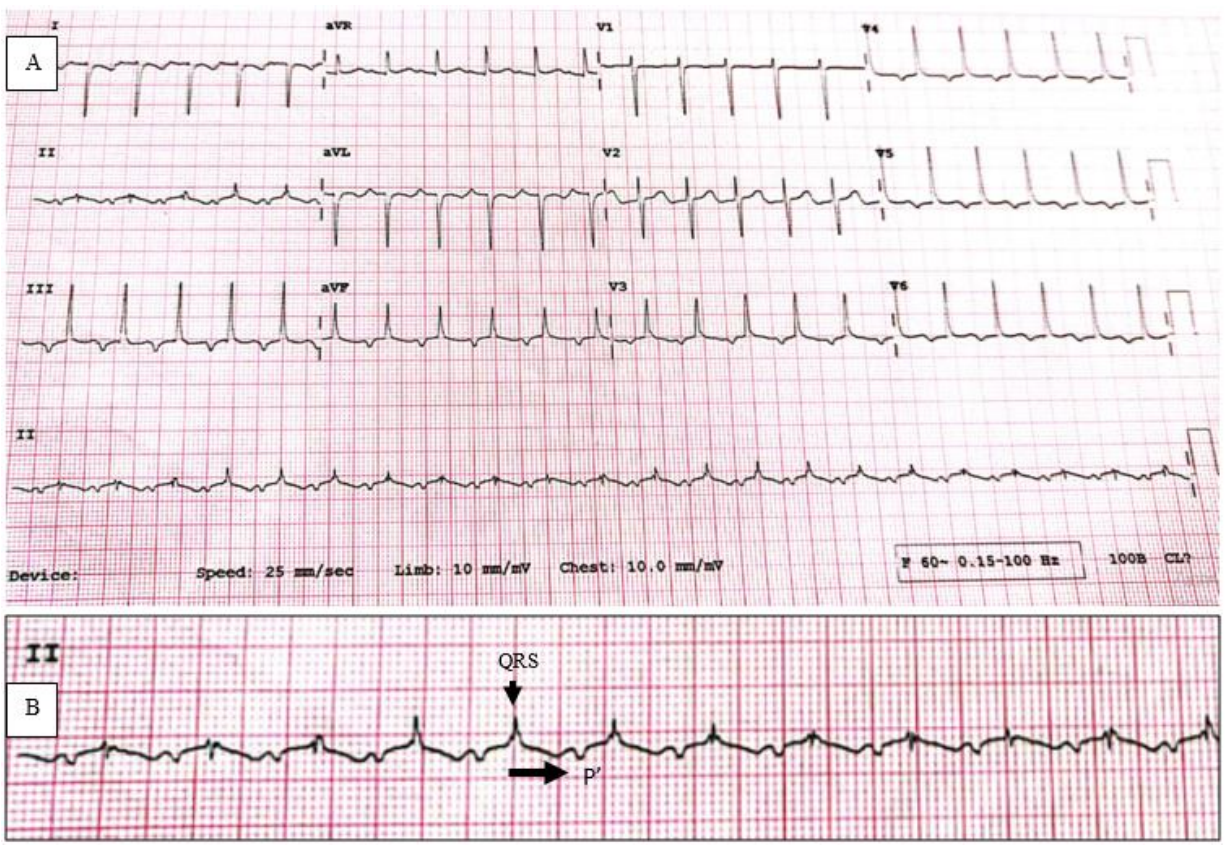

Figure 1A: The 12 lead ECG was done in the ER as the patient came in. The ECG reveals limb lead reversal with QRS being negative in lead I and positive in aVR. The rate was between 140 and 150 bpm. 1B: A "P" wave was visible in limb leads and was inverted in the inferior leads. The RP interval was longer than the PR interval (long RP tachycardia). Additionally, there was respiratory variation in the QRS complexes that is usually seen in a gestational state.
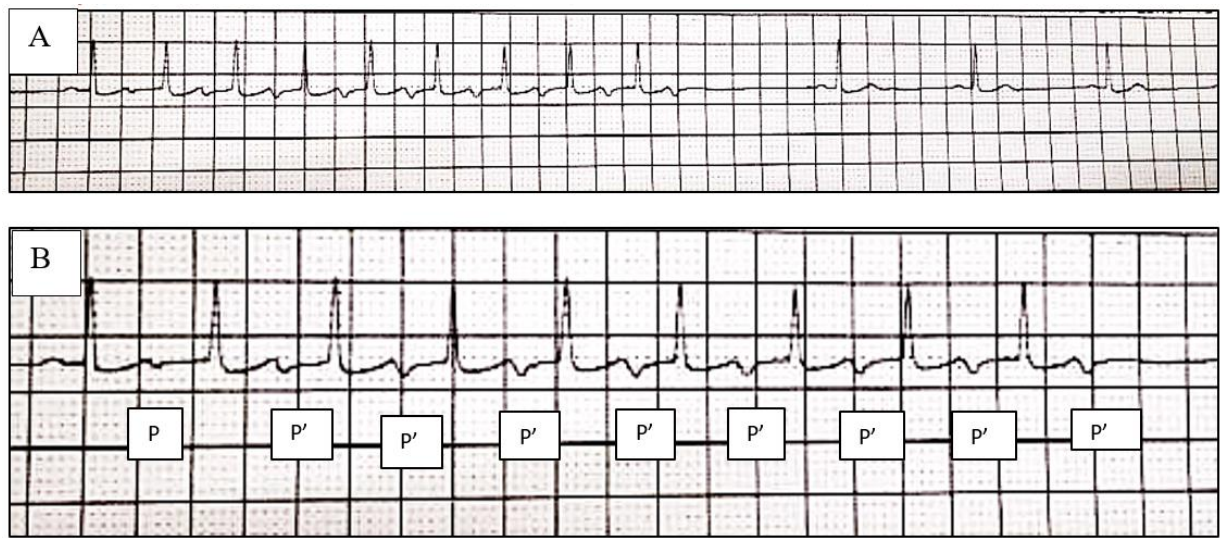

Figure 2A: The first rhythm strip shows lead II from a CCU bed continuous monitor. It shows SVT at $130-140$ bpm, which starts with a "P" wave which appears to be an ectopic premature beat and goes to the QRS via a long PR interval of $240 \mathrm{~ms}$ approximately, which starts a narrow complex tachycardia. The P' waves are retrograde $\mathrm{P}$ waves. The tachycardia terminates on a $\mathrm{P}$ ' wave, which practically ruled out an atrial tachycardia. 


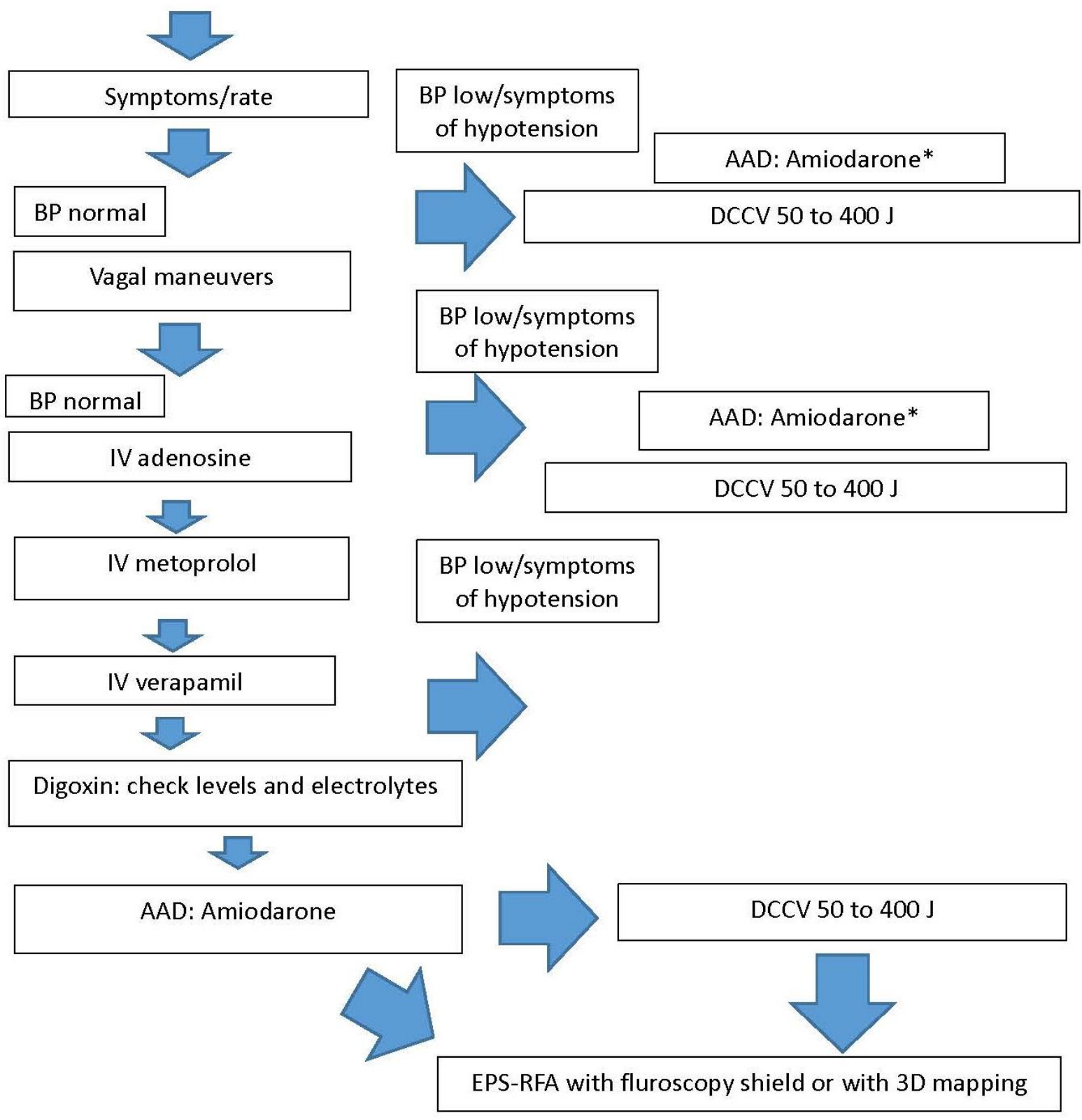

AAD: Antiarrhythmic drugs; DCCV: direct current cardioversion; EPS-RFA: Electrophysiologic study and radiofrequency ablation; * Amiodarone with DCCV for more treatment efficacy

Figure 3: Algorithm of SVT management during gestation

\section{LEARNING POINTS}

1. SVT are common during pregnancy and can have significant hemodynamic effects causing harm to the fetus.

2. Treatment remains the same with vagal maneuvers, adenosine, IV beta blockers and calcium channel blockers as first line therapy.

3. Digoxin has a good safety profile but is less used. It should be remembered as a good second line therapy. It has a narrow therapeutic window but, in that range, a good safety profile during gestation. The drug levels and electrolytes need to be monitored meticulously.

4. Antiarrhythmic drugs like amiodarone to be used as a last resort and only for the acute treatment.

5. In hemodynamically unstable patient or in drug resistant symptomatic SVT, DCCV can be safely used.

6. In resistant and recurrent SVT (that is resistant to all the above measures), catheter ablation can 
be undertaken, with good results. Fetal shield, low level fluoroscopic use or using a 3-D mapping system that can obviate the exposure to radiation but also adds to the cost of the procedure.

\section{REFERENCES}

1. Zipes DP, Libby P, Bonow RO, Braunwald E eds. Braunwald's Heart Disease. 7th edn Philadelphia: Elsevier Saunders, 2005.

2. Carruth JE, Mivis SB, Brogan DR, Wenger NK. The electrocardiogram in normal pregnancy. Am Heart $\mathrm{J}$. 1981;102 (Part 1):1075-8.

3. Rosano GM, Leonardo F, Sarrel PM, Beale CM, De Luca F, Collins P. Cyclical variation in paroxysmal supraventricular tachycardia in women. Lancet. 1996;347:786-8.

4. Greenwood JP, Scott EM, Stoker JB, Walker JJ, Mary DA. Sympathetic neural mechanisms in normal and hypertensive pregnancy in humans. Circulation. 2001;104:2200-4.

5. Cordina R, McGuire MA. Maternal cardiac arrhythmias during pregnancy and lactation. Obstet Med. 2010;3(1):816.

6. Ibetoh CN, Stratulat E, Liu F, Wuni GY, Bahuva R, Shafiq MA, et al. Supraventricular Tachycardia in Pregnancy: Gestational and Labor Differences in Treatment. Cureus. 2021;13(10):e18479.

7. Enriquez AD, Economy KE, Tedrow UB. Contemporary management of arrhythmias during pregnancy. Circ Arrhythm Electrophysiol. 2014;7:961-7.

8. Landy HJ, Weiner S, Corson SL, Batzer FR, Bolognese RJ. The "vanishing twin": ultrasonographic assessment of fetal disappearance in the first trimester. Am J Obstet Gynecol. 1986;155(1):14-9.

9. Katritsis DG, Sepahpour A, Marine JE, Katritsis GD, Tanawuttiwat T, Calkins H, et al. Atypical atrioventricular nodal reentrant tachycardia: prevalence, electrophysiologic characteristics, and tachycardia circuit. Europace. 2015;17:1099-06.

10. Katritsis DG, Josephson ME. Classification, electrophysiological features and therapy of atrioventricular nodal reentrant tachycardia. Arrhythm Electrophysiol Rev. 2016;5(2):130.

11. Li JM, Nguyen C, Joglar JA, Hamdan MH, Page RL. Frequency and outcome of arrhythmias complicating admission during pregnancy: experience from a highvolume and ethnically-diverse obstetric service. Clin Cardiol. 2008;31:538-41

12. Massari F, Scicchitano P, Potenza A, Sassara M, Sanasi M, Liccese M, et al. Supraventricular tachycardia, pregnancy, and water: a new insight in lifesaving treatment of rhythm disorders. Ann Noninvasive Electrocardiol. 2018;23:e12490.

13. Ghosh N, Luk A, Derzko C, Dorian P, Chow C. The acute treatment of maternal supraventricular tachycardias during pregnancy: a review of the literature. J Obstet Gynaecol Can. 2011;33:17-23.

14. Yılmaz F, Beydilli I, Kavalcı C, Yılmaz S. Successful electrical cardioversion of supraventricular tachycardia in a pregnant patient. Am J Case Rep. 2012;13:33-5.

15. Bigelow AM, Crane SS, Khoury FR, Clark JM. Catheter ablation of supraventricular tachycardia without fluoroscopy during pregnancy. Obstet Gynecol. 2015; 125:1338-41.

16. Pires LM, Leiria TL, Kruse ML, de Lima GG. Nonfluoroscopic catheter ablation: a randomized trial. Indian Pacing Electrophysiol J. 2019;19:189-94.

17. Chen G, Sun G, Xu R, Chen X, Yang L, Bai Y, et al. Zerofluoroscopy catheter ablation of severe drug-resistant arrhythmia guided by Ensite NavX system during pregnancy: two case reports and literature review. Medicine (Baltimore). 2016;95:e4487.

18. Yoshimura T, Yoshimura M, Yasue H, Ito M, Okamura H, Mukoyama M, et al. Plasma concentration of atrial natriuretic peptide and brain natriuretic peptide during normal human pregnancy and the postpartum period. J Endocrinol. 1994;140:393-7.

Citation: Ali NF, Qazi SS, Ahmed I, Tamiz MA, Ali A, Khan AH. The Role of Digoxin in the Successful

Termination of a Resistant Maternal Atypical AV Nodal Reentry Tachycardia. Pak Heart J. 2021;54(04):385-389.

DOI: https://doi.org/10.47144/phj.v54i4.2241

Address for Correspondence:

Dr. Aamir Hameed Khan, Professor of Medicine and Consultant Cardiologist at Aga Khan University, Karachi, Pakistan.

Email: aamir.hameed@aku.edu 